\title{
Extrapolations of Lattice Meson Form Factors
}

\author{
T. B. Bunton* F.-J. Jiang $₫$ and B. C. Tiburz \\ Department of Physics \\ Duke University \\ P.O. Box 90305 \\ Durham, NC 27708-0305
}

(Dated: July 30, 2018)

\begin{abstract}
We use chiral perturbation theory to study the extrapolations necessary to make physical predictions from lattice QCD data for the electromagnetic form factors of pseudoscalar mesons. We focus on the quark mass, momentum, lattice spacing, and volume dependence and apply our results to simulations employing mixed actions of Ginsparg-Wilson valence quarks and staggered sea quarks. To determine charge radii at quark masses on the lattices currently used, we find that all extrapolations except the one to infinite volume make significant contributions to the systematic error.
\end{abstract}

PACS numbers: 12.38.Gc, 12.39.Fe

"bunton@phy.duke.edu
fjjjiang@phy.duke.edu
bctiburz@phy.duke.edu

Typeset by REVTEX 


\section{INTRODUCTION}

As computing resources and numerical algorithms improve, first principles determination of hadronic observables will be possible with lattice QCD. In the foreseeable future, these numerical determinations will rely on effective field theories to address systematic errors in lattice data. There has been considerable effort to understand and compute effects from the finite volume of the lattice, the discretization chosen for fermions, and most notably the quark mass dependence of observables. To address systematic error related to the treatment of the fermionic determinant, quenched chiral perturbation theory [1, 2, 3], and partially quenched chiral perturbation theory [4, 5, 6, 6, 8] have been developed. In the respective approximation made in the former case, the determinant is replaced by a constant leading to uncontrolled systematic error, while in the latter the determinant is computed but with larger quark masses used than in the propagators connected to external legs. Only through this latter approximation can connection to real QCD observables be made; moreover, effective field theory is required to make this connection systematically.

In this work we study the extraction of the electromagnetic charge radii of pseudoscalar mesons from lattice QCD within the framework of partially quenched chiral perturbation theory. We consider the various extrapolations in quark mass, momentum, volume and lattice spacing needed to extract the radii. Experimentally, the pion charge radius is rather well determined from pion scattering off atomic electrons [9, 10]. As such it can be used as a crucial test of lattice and effective field theory methods. Away from small momentum transfer, the pion form factor has been experimentally probed from the virtual pion cloud of the nucleon [1] ; however, extraction is limited by model dependence assumed in extrapolating the experimental data to zero virtuality. Ultimately lattice methods will enable first principles QCD calculation of meson form factors over a wide range of momentum transfer.

The original lattice QCD calculations of the pion form factor were pursued by two groups [12, 13]. Since these pioneering calculations there have been various further computations and refinements using improved actions, larger volumes, and different lattice fermions; for recent investigations see, e.g., [14, 15, 16, 17]. These calculations are all limited by the quenched approximation. There have been, however, recent lattice calculations that include dynamical quarks [18, 19]. The results with dynamical quarks in 18] use a mixed lattice action of domain wall valence quarks on staggered sea quarks, and the lightest pion mass $\sim 300 \mathrm{MeV}$ is arguably within the chiral regime. Indeed such mixed action simulations are currently popular due to both the publicly available MILC configurations [20], and the desirable chiral symmetry properties of domain wall fermions [21] (or more generally, of Ginsparg-Wilson fermions 22]). For other observables calculated with such mixed actions, see [23, 24, 25, 26, 27, 28, 29, 30, 31, 32]. The low-energy effective theory for mixed lattice actions is a partially quenched chiral perturbation theory even when the valence and sea quark masses are degenerate. An additional reason for us to study the pion form factor is to learn about the low-energy constants of mixed action chiral perturbation theory 33]. At the order we work, only one new parameter $C_{\text {mix }}$ [see Eq. (21)] enters in the continuum extrapolation of the pion charge radius. We find, however, that the data in [18] allow for only a rough estimate of the parameter $C_{\text {mix }}$, a problem that can be remedied with more lattice data for differing valence quark masses, or at different lattice spacings.

The organization of the paper is as follows. First in Sec. III we review the basics or partially quenched chiral perturbation theory. In Sec. III we derive the meson form factors at one-loop order in partially quenched chiral perturbation theory. Our result improves upon 
an earlier calculation [34] by using a computationally judicious choice for the quark charges. Next in Sec. [V] we include the effects from the lattice discretization for the case of a mixed action of Ginsparg-Wilson valence quarks and staggered sea quarks. The finite volume corrections are presented in Sec. $\nabla$. Extrapolations are considered in Sec. VI, where we use our results to investigate the chiral, momentum, volume, and continuum extrapolations of meson form factors. Lastly we conclude with a brief summary of our work, Sec. VII]

\section{PARTIALLY QUENCHED CHIRAL LAGRANGIAN}

In partially quenched QCD, the quark part of the continuum Lagrangian is written as

$$
\mathcal{L}=\sum_{j=1}^{9} \bar{Q}_{j}\left(\not D+m_{Q}\right) Q_{j}
$$

The nine quarks appear in the vector

$$
Q=(u, d, s, j, l, r, \tilde{u}, \tilde{d}, \tilde{s})^{\mathrm{T}},
$$

that transforms in the fundamental representation of the graded group $S U(6 \mid 3)$ [35, 36]. The quark mass matrix is given by

$$
m_{Q}=\operatorname{diag}\left(m_{u}, m_{d}, m_{s}, m_{j}, m_{l}, m_{r}, m_{u}, m_{d}, m_{s}\right)
$$

to maintain the cancellation of path integral determinants from the valence and ghost sectors. Effects of dynamical quarks are present due to the contribution of the finite-mass sea quarks. Additionally we choose to work in the isospin limit in the valence and sea sectors: $m_{d}=m_{u}$ and $m_{l}=m_{j}$.

The light quark electric charge matrix $\mathcal{Q}$ is not uniquely defined in partially quenched QCD [37]. The only constraint one must impose is that the charge matrix $\mathcal{Q}$ has vanishing supertrace. Following [38, 39], we use

$$
\mathcal{Q}=\operatorname{diag}\left(q_{u}, q_{d}, q_{s}, q_{j}, q_{l}, q_{r}, q_{u}, q_{d}, q_{s}\right)
$$

along with the condition $q_{j}+q_{l}+q_{r}=0$. QCD is recovered in the limit of degenerate valence and sea quarks only for the particular choice: $q_{u}=q_{j}=\frac{2}{3}$, and $q_{d}=q_{s}=q_{l}=q_{r}=-\frac{1}{3}$. Letting the charges be arbitrary, however, enables us to track the flow of charge in loop diagrams.

For massless quarks, the Lagrangian in Eq. (11) exhibits the graded symmetry $S U(6 \mid 3)_{L} \otimes$ $S U(6 \mid 3)_{R} \otimes U(1)_{V}$ that we assume is spontaneously broken to $S U(6 \mid 3)_{V} \otimes U(1)_{V}$. The low-energy effective theory of partially quenched QCD is written in terms of the pseudoGoldstone mesons emerging from spontaneous chiral symmetry breaking. At lowest order in the chiral expansion, the dynamics of these mesons can be described by the $\mathcal{O}\left(p^{2}\right)$ Lagrangian $^{1}$

$$
\mathcal{L}=\frac{f^{2}}{8} \operatorname{str}\left(D_{\mu} \Sigma^{\dagger} D_{\mu} \Sigma\right)-\frac{\lambda}{4} \operatorname{str}\left(m_{Q}^{\dagger} \Sigma+m_{Q} \Sigma^{\dagger}\right)+\alpha \partial_{\mu} \Phi_{0} \partial_{\mu} \Phi_{0}+\mu_{0}^{2} \Phi_{0}^{2}
$$

\footnotetext{
${ }^{1}$ Here $p \sim m_{\pi}$ where $p$ is an external momentum.
} 
where

$$
\begin{aligned}
& \Sigma=\exp \left(\frac{2 i \Phi}{f}\right), \\
& \Phi=\left(\begin{array}{cc}
M & \chi^{\dagger} \\
\chi & \tilde{M}
\end{array}\right),
\end{aligned}
$$

$f=132 \mathrm{MeV}$, and we have defined the electromagnetic gauge-covariant derivative $D_{\mu} \Sigma=$ $\partial_{\mu} \Sigma+i e \mathcal{A}_{\mu}[\mathcal{Q}, \Sigma]$. The $\operatorname{str}()$ denotes a supertrace over flavor indices. The $M, \tilde{M}$, and $\chi$ are matrices of pseudo-Goldstone bosons with quantum numbers of $q \bar{q}$ pairs, pseudo-Goldstone bosons with quantum numbers of $\tilde{q} \bar{q}$ pairs, and pseudo-Goldstone fermions with quantum numbers of $\tilde{q} \bar{q}$ pairs, respectively. Upon expanding the Lagrangian in (5) one finds that quark basis mesons with quark content $Q \bar{Q}^{\prime}$ have masses

$$
m_{Q Q^{\prime}}^{2}=\frac{\lambda}{f^{2}}\left(m_{Q}+m_{Q^{\prime}}\right)
$$

The flavor singlet field appearing above is given by $\Phi_{0}=\operatorname{str}(\Phi) / \sqrt{6}$. Just as in chiral perturbation theory, but in contrast to the quenched case, the singlet field is rendered heavy by the strong $U(1)_{A}$ anomaly and is integrated out of the theory. The resulting flavor neutral propagators, however, deviate from simple pole forms [8]. We do not display these propagators here as they are not explicitly needed in our final results for meson form factors.

Additionally there are three terms in the $\mathcal{O}\left(p^{4}\right)$ Lagrangian

$$
\begin{aligned}
\mathcal{L}= & \alpha_{4} \operatorname{str}\left(D_{\mu} \Sigma D_{\mu} \Sigma^{\dagger}\right) \operatorname{str}\left(m_{Q}^{\dagger} \Sigma+m_{Q} \Sigma^{\dagger}\right)+\alpha_{5} \operatorname{str}\left(D_{\mu} \Sigma D_{\mu} \Sigma^{\dagger}\left(m_{Q}^{\dagger} \Sigma+m_{Q} \Sigma^{\dagger}\right)\right) \\
& -i \alpha_{9} \operatorname{str}\left(L_{\mu \nu} D_{\mu} \Sigma D_{\nu} \Sigma^{\dagger}+R_{\mu \nu} D_{\mu} \Sigma^{\dagger} D_{\nu} \Sigma\right)
\end{aligned}
$$

that contribute to meson form factors at tree level. Here $L_{\mu \nu}, R_{\mu \nu}$ are the field-strength tensors of the external sources, which for an electromagnetic source are given by

$$
L_{\mu \nu}=R_{\mu \nu}=e \mathcal{Q}\left(\partial_{\mu} \mathcal{A}_{\nu}-\partial_{\nu} \mathcal{A}_{\mu}\right)+i e^{2} \mathcal{Q}^{2}\left[\mathcal{A}_{\mu}, \mathcal{A}_{\nu}\right] .
$$

Unlike quenched chiral perturbation theory, where the low-energy constants are distinct from those in chiral perturbation theory, the partially quenched parameters in Eq. (9) are the dimensionless Gasser-Leutwyler parameters of chiral perturbation theory [40], which can be demonstrated by matching.

\section{FORM FACTORS IN INFINITE VOLUME}

The electromagnetic form factor $G_{X}$ of an octet meson $\phi_{X}$ is required by Lorentz invariance and gauge invariance to have the form

$$
\left\langle\phi_{X}\left(p^{\prime}\right)\left|J_{\mu}\right| \phi_{X}(p)\right\rangle=e G_{X}\left(q^{2}\right)\left(p+p^{\prime}\right)_{\mu},
$$

where $p\left(p^{\prime}\right)$ is the momentum of the incoming (outgoing) meson, and $q_{\mu}=\left(p^{\prime}-p\right)_{\mu}$ is the momentum transfer. Conservation of electric charge protects it from renormalization, hence at zero momentum transfer $e G_{X}(0)=Q_{X}$, where $Q_{X}$ is the charge of $\phi_{X}$. The charge radius $r_{X}$ is related to the slope of $G_{X}\left(q^{2}\right)$ at $q^{2}=0$, namely

$$
<r_{X}^{2}>=-\left.6 \frac{d}{d q^{2}} G_{X}\left(q^{2}\right)\right|_{q^{2}=0} .
$$




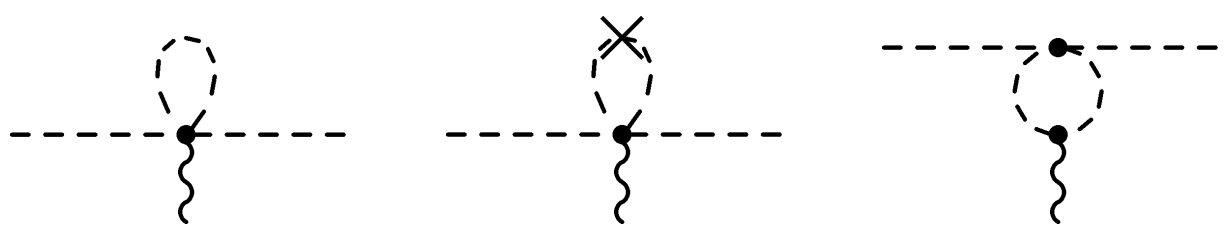

FIG. 1: Loop diagrams contributing to the octet meson charge radii in partially quenched chiral perturbation theory. Octet mesons are denoted by a dashed line, singlets (hairpins) by a crossed dashed line, and the photon by a wiggly line.
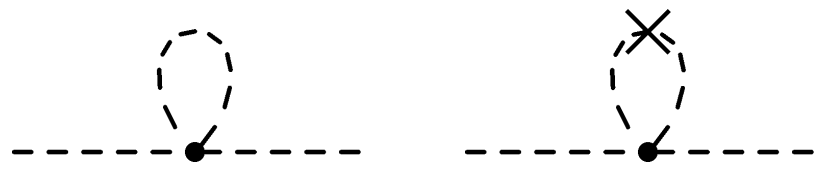

FIG. 2: Wavefunction renormalization diagrams in partially quenched chiral perturbation theory.

Charge conjugation implies the form factor relations: $G_{\pi^{+}}\left(q^{2}\right)=-G_{\pi^{-}}\left(q^{2}\right), G_{K^{+}}\left(q^{2}\right)=$ $-G_{K^{-}}\left(q^{2}\right)$, and $G_{K^{0}}\left(q^{2}\right)=-G_{\bar{K}^{0}}\left(q^{2}\right)$, as well as $G_{\pi^{0}}\left(q^{2}\right)=G_{\eta}\left(q^{2}\right)=0$.

To calculate the charge radii to lowest order in the chiral expansion one has to include operators of $\mathcal{L}$ in Eq. (5) to one-loop order [see Figs. (11) and (2)] and operators of Eq. (9) to tree level. Using dimensional regularization, where our subtraction scheme removes $\frac{1}{\varepsilon}+$ $1-\gamma_{\mathrm{E}}+\log 4 \pi$, we find the form factors have the form

$$
G_{X}\left(q^{2}\right)=Q_{X}\left(1-\frac{4 \alpha_{9}}{f^{2}} q^{2}\right)+\frac{1}{(4 \pi f)^{2}} \sum_{\phi} A_{\phi}^{X} F\left(m_{\phi}^{2}, q^{2}\right) .
$$

In this general expression, $Q_{X}$ is the meson charge, the sum on $\phi$ runs over all non-degenerate loop mesons of mass $m_{\phi}$. The coefficients $A_{\phi}^{X}$ are products of charge and Clebsch-Gordan factors for the coupling of the loop meson $\phi$ to the external state meson $X$. These coefficients are listed in Table I. If a particular loop meson is not listed, then the values of $A_{\phi}^{X}$ are identically zero for all states $X$. Notice that in the isospin limit the charges $q_{j}$ and $q_{l}$ always enter in the combination $q_{j}+q_{l}=-q_{r}$ and for this reason do not explicitly appear in coefficients listed in the table. Lastly the non-analytic function $F\left(m^{2}, q^{2}\right)$ above is defined to be

$$
F\left(m^{2}, q^{2}\right)=\frac{1}{6}\left[q^{2} \log \frac{m^{2}}{\mu^{2}}+4 m^{2} \mathcal{F}\left(\frac{-q^{2}}{4 m^{2}}\right)\right],
$$

where the auxiliary function $\mathcal{F}(a)$ is given by

$$
\mathcal{F}(a)=(a-1) \sqrt{1-\frac{1}{a}} \log \frac{\sqrt{1-\frac{1}{a}+i \varepsilon}-1}{\sqrt{1-\frac{1}{a}+i \varepsilon}+1}+\frac{5}{3} a-2 .
$$

In the limit $m_{j} \rightarrow m_{u}, m_{r} \rightarrow m_{s}$ and using the physical quark charges, we recover the chiral perturbation theory result [40, 41].

Using the values from the Table, the form factor of the $\pi^{+}$, e.g., appears as

$$
G_{\pi^{+}}\left(q^{2}\right)=Q_{\pi^{+}}\left\{1-\frac{4 \alpha_{9}}{f^{2}} q^{2}+\frac{1}{(4 \pi f)^{2}}\left[2 F\left(m_{j u}^{2}, q^{2}\right)+F\left(m_{r u}^{2}, q^{2}\right)\right]\right\},
$$


TABLE I: Coefficients $A_{\phi}^{X}$ of loop mesons contributing to electromagnetic form factors. We list the coefficients only for $X=\pi^{+}, K^{+}$, and $K^{0}$ states because the rest follow from charge conjugation: $A_{\phi}^{\pi^{+}}=-A_{\phi}^{\pi^{-}}, A_{\phi}^{K^{+}}=-A_{\phi}^{K^{-}}$, and $A_{\phi}^{K^{0}}=-A_{\phi}^{\bar{K}^{0}}$ for all $\phi$. And of course $A_{\phi}^{\pi^{0}}=A_{\phi}^{\eta}=0$.

\begin{tabular}{c|cccc}
\hline \hline$X$ & \multicolumn{4}{|c}{$\phi$} \\
& $j u$ & $r u$ & $j s$ & $r s$ \\
\hline$\pi^{+}$ & $2\left(q_{u}-q_{d}\right)$ & $q_{u}-q_{d}$ & 0 & 0 \\
$K^{+}$ & $2 q_{u}+q_{r}$ & $q_{u}-q_{r}$ & $-2 q_{s}-q_{r}$ & $q_{r}-q_{s}$ \\
$K^{0}$ & $2 q_{d}+q_{r}$ & $q_{d}-q_{r}$ & $-2 q_{s}-q_{r}$ & $q_{r}-q_{s}$ \\
\hline \hline
\end{tabular}

where the charge of the pion is $Q_{\pi^{+}}=q_{u}-q_{d}$. The pion form factor appears rather special at one loop due to its independence from the charges of the sea quarks. ${ }^{2}$ In fact, this independence from sea quark charges holds non-perturbatively and was shown using the behavior of lattice correlators under charge conjugation in [13]. We demonstrate this more simply as follows.

The electromagnetic current $J_{\mu}$ can be decomposed into isosinglet and isovector combinations, $J_{\mu}=J_{\mu}^{I}+J_{\mu}^{3}$. Matrix elements of the former involve the charges of the sea quarks; while in the isospin limit, matrix elements of the latter are independent of these sea charges. Now consider the electromagnetic current matrix element of the neutral pion, ${ }^{3}$

$$
\left\langle\pi^{0}\left(P^{\prime}\right)\left|J_{\mu}\right| \pi^{0}(P)\right\rangle=\left\langle\pi^{0}\left(P^{\prime}\right)\left|J_{\mu}^{I}\right| \pi^{0}(P)\right\rangle+\left\langle\pi^{0}\left(P^{\prime}\right)\left|J_{\mu}^{3}\right| \pi^{0}(P)\right\rangle .
$$

This vanishes by charge conjugation invariance. Furthermore the isovector contribution vanishes, hence, so too must the isosinglet contribution. The matrix element of the isosinglet current is the same for all pions in the isospin limit. Thus as a consequence of isospin symmetry and charge conjugation invariance, we have

$$
\left\langle\pi^{+}\left(P^{\prime}\right)\left|J_{\mu}\right| \pi^{+}(P)\right\rangle=\left\langle\pi^{+}\left(P^{\prime}\right)\left|J_{\mu}^{3}\right| \pi^{+}(P)\right\rangle,
$$

and hence the charged pion form factor is independent of the sea quark charges.

As a result of this independence from sea quark charges, one can efficaciously ignore operator self-contractions and still determine the pion form factor [13]. Note that while the pion form factor is insensitive to contributions from closed quark loops with photon insertion, it is sensitive to sea quarks. As pointed out in [34], the analogous calculation in quenched chiral perturbation theory shows that there is no meson mass dependence at one-loop order. This result is also clear from the Table: only valence-sea loop mesons enter our one-loop expressions. Notice that the kaon form factors are not independent from the

\footnotetext{
${ }^{2}$ At one loop, we can see explicitly that the independence from sea quark charges arises from cancellations between isospin degenerate loop mesons. Away from the isospin limit the sea charges remain, e.g., in non-degenerate $S U(4 \mid 2)$ the non-vanishing loop coefficients for the $\pi^{+}$are: $A_{j u}^{\pi^{+}}=q_{u}-q_{j}, A_{l u}^{\pi^{+}}=q_{u}-q_{l}$, $A_{j d}^{\pi^{+}}=-\left(q_{d}-q_{j}\right)$, and $A_{l d}^{\pi^{+}}=-\left(q_{d}-q_{l}\right)$.

${ }^{3}$ As with the proof in [13], our argument holds for charged $S U(2)$ mesons of arbitrary spin and parity in the isospin limit, e.g. one can use the charge conjugation invariance of the $\rho^{0}$ to establish that the form factor of the $\rho^{+}$is independent of the charges of the sea.
} 
sea quark charges. This dependence only disappears in the $S U(3)$ limit, which is badly violated in nature. ${ }^{4}$

Finally we derive expressions for the charge radii at one-loop order. In infinite volume, we can take $q^{2} \ll m^{2}$ to find that the meson charge radii are given by

$$
<r_{X}^{2}>=Q_{X} \frac{24 \alpha_{9}}{f^{2}}-\frac{1}{(4 \pi f)^{2}} \sum_{\phi} A_{\phi}^{X}\left(\log \frac{m_{\phi}^{2}}{\mu^{2}}+1\right) \text {. }
$$

\section{LATTICE SPACING DEPENDENCE}

In this Section, we detail the modifications to our results at finite lattice spacing. We consider a mixed lattice action consisting of Ginsparg-Wilson valence quarks and staggered sea quarks. To address the effects of the lattice spacing, one formulates the continuum effective theory of the lattice action and then matches this effective theory onto a chiral perturbation theory. In this work we assume the natural hierarchy of scales

$$
m_{q} \ll \Lambda_{\mathrm{QCD}} \ll \frac{1}{a},
$$

and choose the power counting

$$
p^{2} \sim m_{\pi}^{2} \sim a^{2} \Lambda_{\mathrm{QCD}}^{4}
$$

The form factors can now be systematically calculated in the dual expansion in quark mass and lattice spacing. Such modifications to the electromagnetic form factors of mesons are rather simple. It was demonstrated in Ref. [42] that no local $a$-dependent operators contribute to the form factors to $\mathcal{O}\left(p^{2}\right)$ due to charge conservation. In our power counting, local corrections to the current in the $a^{2}$ chiral Lagrangian will contribute at $\mathcal{O}\left(p^{4}\right)$ and will be competitive with two-loop effects. These local terms can thus be neglected here. The lattice spacing corrections to meson form factors then enter only through the dependence of the meson masses on the lattice spacing.

With a mixed lattice action, there is no symmetry that relates the valence and sea sectors of the theory. The Symanzik Lagrangian thus contains dimension-six mixed field bilinears of the form [33, 43.

$$
\delta \mathcal{L}^{(6)} \sim\left(\bar{Q} \Gamma P_{V} Q\right)\left(\bar{Q} \Gamma P_{S} Q\right)
$$

where $P_{V}$ is a diagonal matrix that has unit entries corresponding to the valence sector, and $P_{S}$ is a diagonal matrix that has unit entries corresponding to the sea sector (we have also implicitly enlarged the vector $Q$ to transform under the continuum $S U(15 \mid 3)$ that includes four tastes for each flavor of sea quark). Above $\Gamma=\gamma_{\mu}, \gamma_{\mu} \gamma_{5}$ are the only Dirac matrices allowed by the chiral symmetry of the valence sector and the axial symmetry of the sea sector. The mapping of such mixed bilinears onto operators in the chiral perturbation theory at finite lattice spacing produces one new operator of the form 33 ]

$$
\delta \mathcal{L}=-\frac{1}{8} a^{2} C_{\text {mix }} \operatorname{str}\left(T_{3} \Sigma T_{3} \Sigma^{\dagger}\right)
$$

\footnotetext{
${ }^{4}$ Indeed the argument presented in [13] only applies for mesons consisting of degenerate flavors. In the non-degenerate case, the argument reduces to a demonstration that the sum of operator self-contractions in the meson and its charge conjugate vanish.
} 
where $T_{3}=P_{S}-P_{V}$. This operator contributes to masses of mesons formed from one valence and one sea quark. Such valence-sea mesons are not protected from additive mass renormalization due to the mixed action's symmetry. Thus to $\mathcal{O}\left(p^{2}\right)$, the loop meson consisting of a staggered quark $Q_{i}$, i.e. of flavor $Q$ and taste $i$, and a valence quark $Q^{\prime}$ have masses given by [33]

$$
m_{Q_{i} Q^{\prime}}^{2}=\frac{\lambda}{f^{2}}\left(m_{Q}+m_{Q^{\prime}}\right)+\frac{a^{2}}{f^{2}} C_{\text {mix }} .
$$

These masses are independent of the staggered quark taste and hence there is a four-fold taste degeneracy in the valence-sea meson loops. This four-fold degeneracy is exactly canceled by the $\frac{1}{4}$ factor that must be inserted by hand to implement the fourth-root trick. Hence in a mixed action simulation, the meson form factors at $\mathcal{O}\left(p^{2}\right)$ are given by Eq. (13) with the loop meson masses given in Eq. (22).

\section{VOLUME DEPENDENCE}

On the lattice, the available momentum modes are quantized and observables calculated thus inherit a dependence on the lattice volume. This dependence, which is inherently a longdistance effect, can be ascertained using chiral perturbation theory. We customarily choose a hypercubic box of three equal spatial dimensions $L$, and time dimension $T$, with $T \gg L$. With periodic boundary conditions on the quark fields, the available meson momenta have the form $k_{\mu}=\left(k_{0}, \boldsymbol{k}\right)$, with $\boldsymbol{k}=\frac{2 \pi}{L} \boldsymbol{n}$, and $\boldsymbol{n}$ represents a triplet of integers. Due to the assumed length of the time direction, we treat $k_{0}$ as continuous.

As spontaneous symmetry breaking does not occur in finite volumes, we must be careful also to specify that $m_{\pi} L>1$ so that mesonic zero modes do not become strongly coupled [44]. This restriction ensures that the zero modes do not conspire to restore chiral symmetry. Provided this is the case, the standard $p$-counting of chiral perturbation theory remains intact and integrals over loop momenta can merely be replaced by corresponding sums over quantized momenta. This replacement leads to a dependence of physical quantities on the lattice size $L$.

Calculation of the finite volume correction to our one-loop results for meson form factors is straightforward. Considering pionic matrix elements of the charge density ${ }^{5}$ in a box, we have

$$
\begin{aligned}
\frac{\left\langle\phi_{X}\left(p^{\prime}\right)\left|J_{4}\right| \phi_{X}(p)\right\rangle}{p_{4}^{\prime}+p_{4}}= & e Q_{X}\left(1-\frac{4 \alpha_{9}}{f^{2}} q^{2}\right) \\
& +\sum_{\phi, \boldsymbol{k}} \frac{e A_{\phi}^{X}}{2 f^{2} L^{3}} \int_{0}^{1} d x\left[\frac{1}{\sqrt{(\boldsymbol{k}+x \boldsymbol{q})^{2}+m_{\phi}^{2}+x(1-x) q^{2}}}-\frac{1}{\sqrt{\boldsymbol{k}^{2}+m_{\phi}^{2}}}\right] .
\end{aligned}
$$

The finite volume shift can then be obtained using the Poisson re-summation formula and the resulting sums can be cast into exponentially convergent forms, see, e.g. [45]. We find

\footnotetext{
${ }^{5}$ We do this only for simplicity. The form factors can be extracted from any component of the current, and this is the one commonly chosen in lattice QCD simulations. The volume effects from extracting form factors from the spatial components can similarly be calculated.
} 


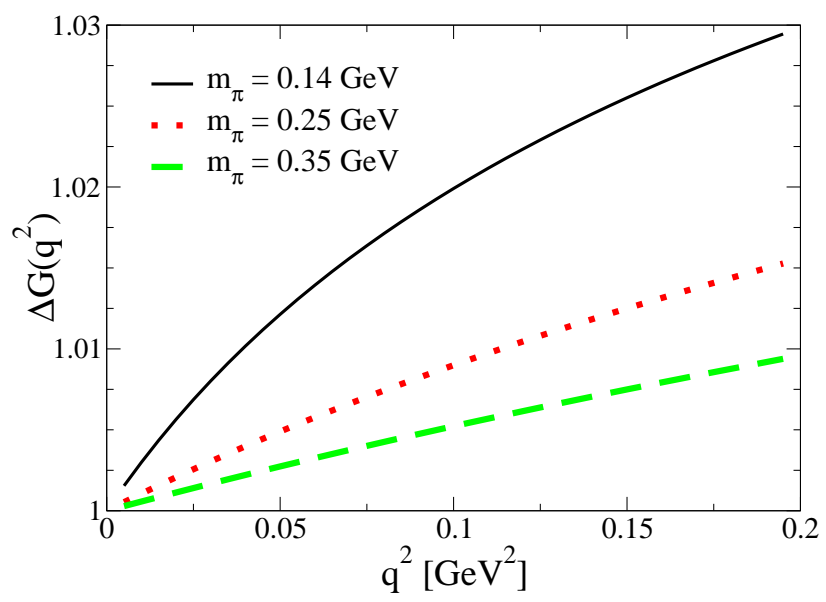

FIG. 3: Momentum transfer dependence of the pion form factor. The ratio of the pion form factor to its slope at zero momentum transfer is plotted versus $q^{2}$ for a range of pion masses.

the finite volume shift to the meson form factors

$$
\delta_{L} G_{X}\left(q^{2}\right)=\frac{1}{(4 \pi f)^{2}} \sum_{\phi} A_{\phi}^{X} \int_{0}^{1} d x\left[\mathcal{I}\left(x \boldsymbol{q}, m_{\phi}^{2}+x(1-x) q^{2}\right)-\mathcal{I}\left(\mathbf{0}, m_{\phi}^{2}\right)\right]
$$

where we have defined

$$
\mathcal{I}(\boldsymbol{q}, \Delta)=\int_{0}^{\infty} d \tau \frac{e^{-\tau \Delta}}{\tau^{2}}\left[\prod_{j=1}^{3} \vartheta_{3}\left(\frac{q_{j} L}{2}, e^{-\frac{L^{2}}{4 \tau}}\right)-1\right]
$$

with $\vartheta_{3}(q, z)$ as the Jacobi elliptic theta function of the third kind.

\section{EXTRAPOLATIONS OF THE CHARGE RADII}

We now consider each of the various extrapolations necessary to make physical predictions from lattice data for meson form factors. For simplicity we consider the pion form factor and this will enable us to compare with the lattice data in [18]. To perform our analysis, we fix the low-energy constant $\alpha_{9}(\mu=1 \mathrm{GeV})=0.0069$ by using the one-loop result Eq. (19) along with the experimentally determined pion charge radius [10].

\section{A. Momentum Extrapolation}

First we shall deal with the limitation of discrete lattice momenta. Above we have determined the effect of the finite volume on the radii by using the allowed lattice momenta in a periodic box. Another consequence of periodic boundary conditions is that the momentum transfer is quantized; hence, the limit leading to Eq. (19) cannot be taken. Provided the lowest momentum transfers are in the chiral regime, the effective field theory can be used to perform a momentum extrapolation, $c f$. Eq. (16). It is questionable whether at current lattice volumes the minimum spatial momentum $\left|\boldsymbol{q}_{\text {min }}\right| \approx 0.5 \mathrm{GeV}$ meets this restriction. For

a relativistic object like the pion, a slight reduction is seen because $q^{2}<\boldsymbol{q}^{2}$ (unlike the 


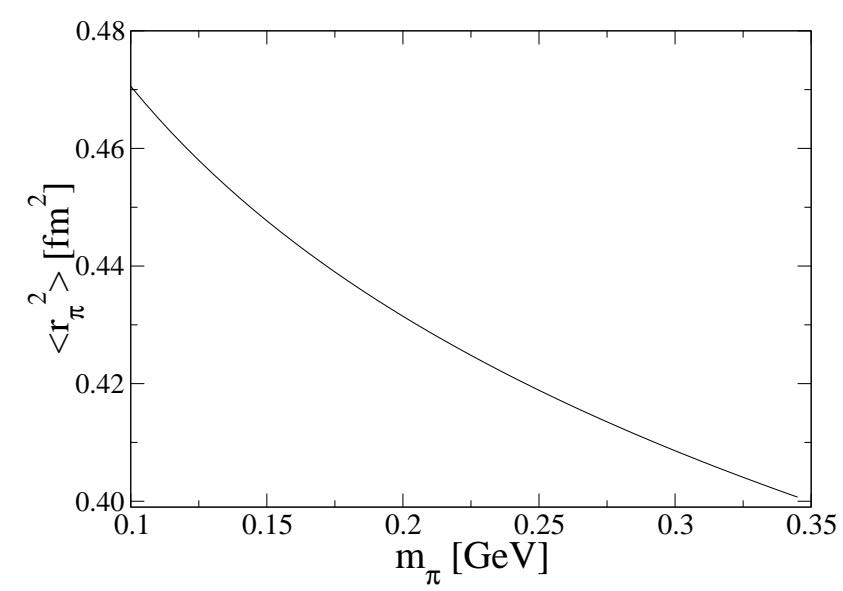

FIG. 4: Meson mass dependence of the pion charge radius.

nucleon where $q^{2} \approx \boldsymbol{q}^{2}$ ). Furthermore, corrections to Eq. (16) from higher terms in the chiral expansion are of order $q^{2} / \Lambda_{\chi}^{2}$, for which the smallest available $q^{2}$ in Ref. [18] yields $\sim 20 \%$ corrections. Thus we shall assume that the momentum transfer dependence of the lattice data at the minimal value of $q^{2}$ is captured by the effective field theory.

With this assumption, let us investigate the momentum transfer dependence of the pion form factor at one-loop order. If the minimal momentum transfer $q^{2}$ is indeed small compared to $m^{2}$, then the form factor will have linear behavior in $q^{2}$ and no momentum extrapolation is necessary to determine the slope near $q^{2}=0$. On the other hand, imagine that $m^{2}$ is small compared to $q^{2}$. In this limit, the chiral logarithm dominates the form factor, which is multiplied by $q^{2}$, and again the behavior is linear. In the intermediate region $q^{2} \sim 4 m^{2}$ the behavior of the function $\mathcal{F}\left[-q^{2} /\left(4 m^{2}\right)\right]$ in Eq. (15) becomes important. This is the region of parameters relevant for current lattice simulations. In Figure 3. we plot the function $\Delta G\left(q^{2}\right)$, defined by

$$
\Delta G\left(q^{2}\right)=\frac{G_{\pi}\left(q^{2}\right)-Q_{\pi}}{q^{2} G_{\pi}^{\prime}\left(q^{2}\right)},
$$

as a function of $q^{2}$ in order to see the deviation from linearity. The largest deviation from linearity over this range of momentum transfer is for the physical pion mass. For the larger pion masses employed on the lattice, the plot shows that for all practical purposes we can treat the form factor as linear in $q^{2}$. For example, the data at the lowest $q_{\text {min }}^{2}$ and at lightest pion mass in Ref. [18], the difference from linearity is less than one percent. Comparatively, the neglected higher-order terms in the chiral expansion are more than an order of magnitude larger (these terms, however, modify the momentum transfer dependence).

\section{B. Chiral Extrapolation}

Most striking is the well-known behavior of the charge radii near the chiral limit. This can be seen from the chiral logarithm in Eq. (19). For arbitrarily light pions the virtual cloud of pionic excitations in a meson extends arbitrarily far, and hence there is considerable variation of the charge radius as a function of the pion mass. One must be careful, however, not to assume that such variation with mass will necessarily be seen in lattice data. Looking at Eqs. (19), and (22), we see that the valence-sea meson mass is what dominates the chiral 


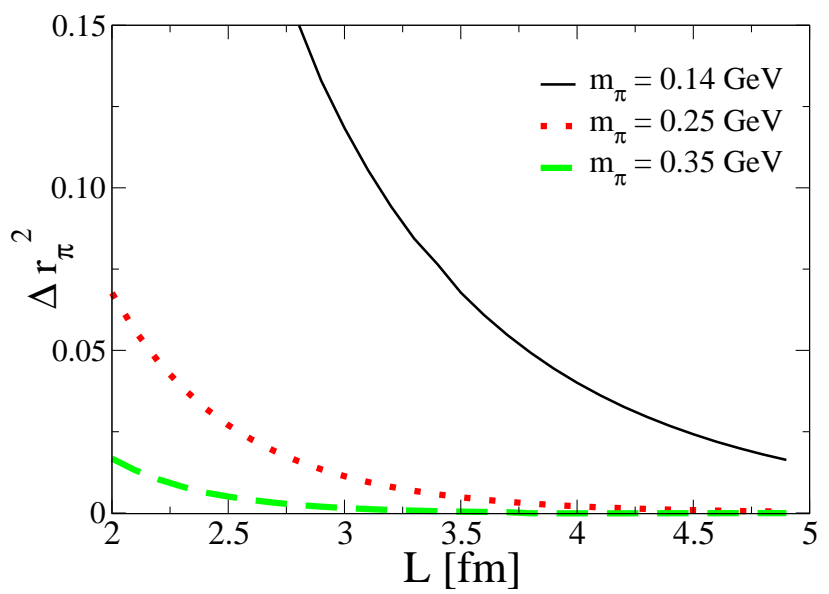

FIG. 5: Volume dependence of the pion charge radius. The relative difference $\Delta r_{\pi}^{2}$ of the charge radius in finite to infinite volume is plotted as a function of the lattice size $L$. The curve for $m_{\pi}=0.14 \mathrm{GeV}$ terminates for $m_{\pi} L=2$ where pionic zero modes become important.

behavior at one-loop order. Thus while the valence-valence and sea-sea meson masses are light, the additive mass renormalization allowed by the mixed-action symmetry breaking may actually push one away from the chiral regime. In Figure 4, we plot the meson mass dependence of the pion charge radius. Keep in mind that the meson mass which is relevant is the valence-sea mass. Ignoring other systematic errors, lattice calculations with valence-sea meson masses $\sim 350 \mathrm{MeV}$ will undershoot the pion charge radius by $\sim 10 \%$.

\section{Volume Extrapolation}

The virtual pion cloud will be affected by the boundary conditions imposed in the lattice simulation. Due to the chiral singularity in the one-loop contribution, one might also expect that the volume effects are substantial for light pions because these corrections stem from the long distance physics. Using Eq. (23) we can plot the volume effect as a function of $L$ for various values of the (valence-sea) pion mass. In Figure 5 , this is done for the quantity $\Delta r_{\pi}^{2}$ which is defined by

$$
\Delta r_{\pi}^{2}=\frac{<r_{\pi}^{2}>_{L}}{<r_{\pi}^{2}>},
$$

where $\left\langle r_{\pi}^{2}>\right.$ is the infinite volume radius, which is given in Eq. (19), and $<r_{\pi}^{2}>_{L}=$ $-\left.6 \frac{d}{d q^{2}} \delta_{L} G_{\pi}\left(q^{2}\right)\right|_{q^{2}=0}$ is the finite volume modification. We see that at the physical value of the pion mass, volume effects are substantial $\sim 12 \%$ in a $3 \mathrm{fm}$ box consistent with our intuition. However, the volume effects drop considerably for larger pion masses. For an $m=0.35 \mathrm{GeV}$ valence-sea pion in a $2.5 \mathrm{fm}$ box [which roughly corresponds to the values used in [18] ignoring the effects of the mixed action in Eq. (22)], the effects of periodic boundary conditions in a finite box lead to a bigger positively charged pion, but by only a negligible $0.5 \%$. 


\section{Continuum Extrapolation}

Lastly we investigate the systematic error in the pion form factor data due to the finite size of the lattice spacing. The lattice spacing $a$ enters our expressions only through the valence-sea meson masses, Eq. (22). Ideally we would have data at multiple values of the lattice spacing and quark mass to enable a proper continuum and chiral extrapolation of the data. In Ref. [18], there is only one lattice spacing employed and only one valence-valence meson mass light enough to warrant a chiral analysis. As we have commented above, volume and momentum extrapolations produce negligible corrections compared to neglected higherorder chiral contributions. Thus we need only use the infinite volume expression in Eq. (16) to make contact with the data. Fixing $\alpha_{9}$ and $f$ to their physical values, we can estimate the mixed action low-energy constant $C_{\text {mix }}$.

Using the form factor data at the lowest value of $q_{\min }^{2}=0.18 \mathrm{GeV}^{2}$ with the valence pion mass $m_{\pi}=0.32 \mathrm{GeV}$ and the sea pion mass $m_{j j}=0.35 \mathrm{GeV}$, we estimate $C_{\text {mix }}=0.0064 \mathrm{GeV}^{6}$. We will not cite errors on this value because it is a rough estimate - only one datum is used to determine $C_{\mathrm{mix}}$. Moreover as it enters our expressions logarithmically, any error in the form factor exponentiates into our estimate of $C_{\text {mix }}$. Using the statistical error bars from the lattice data for a high-low estimate, we find a rather wide range statistically allowed for $C_{\text {mix }}$ from -0.0040 to 0.080 in units of $\mathrm{GeV}^{6}$. The range produced from the systematic uncertainty is comparable.

\section{SUMMARY}

Above we have investigated various extrapolations necessary to connect lattice QCD data for meson form factors at small momentum transfer to the physical meson charge radii. We find that while volume effects are sizable at the physical pion mass, they are negligible for $m_{\pi} \sim 250-350 \mathrm{MeV}$ in current lattice volumes. Provided the minimum lattice

momentum $q_{\min } \lesssim 300 \mathrm{MeV}$, there is no need for a momentum extrapolation: the small $q^{2}-$ dependence predicted from chiral perturbation theory is very linear. The corrections to this linearity arise from next-to-next-to-leading order contributions in the chiral expansion. At these pion masses and momenta, we thus conclude that systematic error is dominated by higher-order terms in the chiral expansion: $\sim 10 \%$ from corrections that scale generically as $m_{\pi}^{2} / \Lambda_{\chi}^{2}$, and $\sim 20 \%$ from terms of order $q^{2} / \Lambda_{\chi}^{2}$ with $q^{2}=q_{\min }^{2}$ of [18]. For the mixed action data in [18], there are additional errors from lattice spacing artifacts. Demanding that their data be described within the effective field theory, we are able to estimate the lowenergy constant $C_{\text {mix }}$ that parametrizes the explicit breaking of the mixed action symmetry $[S U(6 \mid 3) \rightarrow S U(3 \mid 3) \otimes S U(3)]$ at $\mathcal{O}\left(a^{2}\right)$. We obtained a rough value $C_{\text {mix }} \approx 0.006 \mathrm{GeV}^{6}$, where there is sizable error in this value arising from the statistical error in the lattice data and systematic error due to neglecting of two-loop results (for both the quark mass and momentum dependence of the pion form factor). Because $C_{\text {mix }}$ enters the expression for the form factor through a logarithm, a small $\sim 10 \%$ statistical or systematic error in the lattice data results in a very large uncertainty for $C_{\text {mix }}$. Data at multiple lattice spacings or with varying quark mass would be ideal for a better determination of this low-energy constant. Nonetheless, the pion charge radius is a quantity that is sensitive to the fermion discretization, and can be used to understand the errors associated with the continuum extrapolation of mixed-action lattice data.

The computation of meson form factors on the lattice is challenging. For the pion, 
however, the absence of operator self-contractions in the isospin limit [13], puts the charge radius in reach of current lattice technology. Further data at additional values of the valence quark mass and lattice spacing will thus allow one to predict the pion charge radius at about the $\sim 10-20 \%$ level or better, without any model assumptions.

\section{Acknowledgments}

We would like to thank A. Walker-Loud for initial involvement, and R. Lewis for providing the lattice data from [18]. B.C.T. acknowledges the Institute for Nuclear Theory at the University of Washington for hospitality and partial support during the completion of this work. This work is supported in part by the U.S. Department of Energy under Grant No. DE-FG02-05ER41368-0.

[1] A. Morel, J. Phys. (France) 48, 1111 (1987).

[2] S. R. Sharpe, Phys. Rev. D46, 3146 (1992), hep-lat/9205020.

[3] C. W. Bernard and M. F. L. Golterman, Phys. Rev. D46, 853 (1992), hep-lat/9204007.

[4] C. W. Bernard and M. F. L. Golterman, Phys. Rev. D49, 486 (1994), hep-lat/9306005.

[5] S. R. Sharpe, Phys. Rev. D56, 7052 (1997), hep-lat/9707018.

[6] M. F. L. Golterman and K.-C. Leung, Phys. Rev. D57, 5703 (1998), hep-lat/9711033.

[7] S. R. Sharpe and N. Shoresh, Phys. Rev. D62, 094503 (2000), hep-lat/0006017.

[8] S. R. Sharpe and N. Shoresh, Phys. Rev. D64, 114510 (2001), hep-lat/0108003.

[9] S. R. Amendolia et al. (NA7), Nucl. Phys. B277, 168 (1986).

[10] S. Eidelman et al. (Particle Data Group), Phys. Lett. B592, 1 (2004).

[11] J. Volmer et al. (The Jefferson Lab F(pi)), Phys. Rev. Lett. 86, 1713 (2001), nucl-ex/0010009.

[12] G. Martinelli and C. T. Sachrajda, Nucl. Phys. B306, 865 (1988).

[13] T. Draper, R. M. Woloshyn, W. Wilcox, and K.-F. Liu, Nucl. Phys. B318, 319 (1989).

[14] Y. Nemoto (RBC), Nucl. Phys. Proc. Suppl. 129, 299 (2004), hep-lat/0309173.

[15] J. van der Heide, J. H. Koch, and E. Laermann, Phys. Rev. D69, 094511 (2004), heplat/0312023.

[16] A. M. Abdel-Rehim and R. Lewis, Phys. Rev. D71, 014503 (2005), hep-lat/0410047.

[17] S. Capitani, C. Gattringer, and C. B. Lang (Bern-Graz-Regensburg (BGR)), Phys. Rev. D73, 034505 (2006), hep-lat/0511040.

[18] F. D. R. Bonnet, R. G. Edwards, G. T. Fleming, R. Lewis, and D. G. Richards (Lattice Hadron Physics), Phys. Rev. D72, 054506 (2005), hep-lat/0411028.

[19] S. Hashimoto et al. (JLQCD), PoS LAT2005, 336 (2006), hep-lat/0510085.

[20] C. W. Bernard et al., Phys. Rev. D64, 054506 (2001), hep-lat/0104002.

[21] D. B. Kaplan, Phys. Lett. B288, 342 (1992), hep-lat/9206013.

[22] M. Luscher, Phys. Lett. B428, 342 (1998), hep-lat/9802011.

[23] D. B. Renner et al. (LHP), Nucl. Phys. Proc. Suppl. 140, 255 (2005), hep-lat/0409130.

[24] K. C. Bowler, B. Joo, R. D. Kenway, C. M. Maynard, and R. J. Tweedie (UKQCD), JHEP 08, 003 (2005), hep-lat/0411005.

[25] S. R. Beane, P. F. Bedaque, K. Orginos, and M. J. Savage (NPLQCD), Phys. Rev. D73, 054503 (2006), hep-lat/0506013. 
[26] R. G. Edwards et al. (LHPC), PoS LAT2005, 056 (2006), hep-lat/0509185.

[27] C. Alexandrou et al., PoS LAT2005, 091 (2006), hep-lat/0509140.

[28] R. G. Edwards et al. (LHPC), Phys. Rev. Lett. 96, 052001 (2006), hep-lat/0510062.

[29] S. R. Beane, P. F. Bedaque, K. Orginos, and M. J. Savage (2006), hep-lat/0602010.

[30] S. R. Beane, K. Orginos, and M. J. Savage (2006), hep-lat/0604013.

[31] S. R. Beane, K. Orginos, and M. J. Savage (2006), hep-lat/0605014.

[32] S. R. Beane, P. F. Bedaque, K. Orginos, and M. J. Savage (2006), hep-lat/0606023.

[33] O. Bar, C. Bernard, G. Rupak, and N. Shoresh, Phys. Rev. D72, 054502 (2005), heplat/0503009.

[34] D. Arndt and B. C. Tiburzi, Phys. Rev. D68, 094501 (2003), hep-lat/0307003.

[35] A. B. Balantekin and I. Bars, J. Math. Phys. 22, 1149 (1981).

[36] A. B. Balantekin and I. Bars, J. Math. Phys. 23, 1239 (1982).

[37] M. Golterman and E. Pallante, JHEP 10, 037 (2001), hep-lat/0108010.

[38] B. C. Tiburzi, Phys. Rev. D71, 054504 (2005), hep-lat/0412025.

[39] W. Detmold and C. J. D. Lin, Phys. Rev. D71, 054510 (2005), hep-lat/0501007.

[40] J. Gasser and H. Leutwyler, Nucl. Phys. B250, 465 (1985).

[41] J. Gasser and H. Leutwyler, Nucl. Phys. B250, 517 (1985).

[42] D. Arndt and B. C. Tiburzi, Phys. Rev. D69, 114503 (2004), hep-lat/0402029.

[43] O. Bar, G. Rupak, and N. Shoresh, Phys. Rev. D70, 034508 (2004), hep-lat/0306021.

[44] J. Gasser and H. Leutwyler, Phys. Lett. B188, 477 (1987).

[45] C. T. Sachrajda and G. Villadoro, Phys. Lett. B609, 73 (2005), hep-lat/0411033. 